\title{
Integration of dual intelligent algorithms in shunt active power filter
}

\begin{abstract}
This paper presents an integration of dual intelligent algorithms: artificial neural network (ANN) based fundamental component extraction algorithm and fuzzy logic based DC-link voltage self-charging algorithm (fuzzy self-charging algorithm), in a three-phase three-wire shunt active power filter (SAPF). The ANN and fuzzy logic are trained using the modified Widrow-Hoff (W-H) weight updating algorithm and fuzzy rules respectively. The controller has utilized both algorithms for producing its reference signals and consequently mitigating distorted source currents, improving system power factor (PF) and regulating the DC-link voltage. A $10 \mathrm{kHz}$ pulse-width modulation (PWM) switching algorithm is employed to generate the switching signals and therefore the distorted source currents are reshaped to be sinusoidal source current with low total harmonic distortion (THD) value and in phase with source voltages. The effectiveness of the proposed dual intelligent algorithms is verified using Matlab/Simulink.
\end{abstract}

Keyword: Shunt active power filter (SAPF); Artificial neural network (ANN); Harmonic extraction; Current harmonics; Reactive power compensation; Fuzzy logic; DC-link voltage regulation 